\title{
La eficacia clínica de la carga convencional, precoz e inmediata en sobredentaduras mandibulares con implantes dentales. Un estudio comparativo a cinco años
}

\section{The clinical efficacy of conventional, early and immediate in mandibular overdentures with dental implants. $A$ 5-year comparative study}

\author{
LINARES GANCEDO D* \\ MEDEL SOTERAS R** \\ SEGURA EGEA JJ** \\ POYATO FERRERA $\mathbf{M} * * *$ \\ VELASCO ORTEGA $\mathbf{E} * * * *$
}

\begin{abstract}
Linares Gancedo D, Medel Soteras R, Segura Egea JJ, Poyato Ferrera M, Velasco Ortega E. La eficacia clínica de la carga convencional, precoz e inmediata en sobredentaduras mandibulares con implantes dentales. Un estudio comparativo a cinco años. Av Periodon Implantol. 2010; 22, 1: 11-18
\end{abstract}

\section{RESUIMEN}

Introducción. El estudio muestra la evaluación de los pacientes edéntulos totales tratados con sobredentaduras mandibulares con implantes dentales mediante diferentes tipos de carga funcional. Métodos. 33 pacientes edéntulos totales fueron tratados con 127 implantes en la mandíbula para su rehabilitación prostodóncica con sobredentaduras. Todos los implantes fueron insertados en la cirugía y fueron cargados de forma convencional, precoz o inmediata.

Resultados. Después de un seguimiento clínico medio de 67,5 meses (rango: 36-108 meses), los resultados indican un éxito de los implantes del $96,9 \%$ porque 4 implantes fracasaron. El 100\% de los pacientes fueron tratados con una sobredentadura implantosoportada, el 60,9\% con retenedores de bolas y el $31,9 \%$ mediante barras.

Conclusiones. El estudio indica que la rehabilitación oral de los pacientes edéntulos con sobredentaduras mandibulares implantosoportadas mediante su carga convencional, precoz o inmediata pueden obtener la oseointegración y representar una terapéutica odontológica con éxito.

PALABRAS CLAVE: Carga convencional, carga precoz, carga inmediata, sobredentaduras mandibulares, oseointegración, implantología oral.

\section{SUMIMARY}

Introduction. This study reports the evaluation of edentulous patients treated with overdentures in the mandible by different loading protocols of dental implants.

Patients and Methods. 33 edentulous patients were treated with 127 implants for prosthodontic rehabilitation with overdentures in the mandible. All implants were inserted in one-stage and were loaded with conventional, early and immediate protocols.

Results. After a mean follow-up of 67,5 months (range: 36-108 months), clinical results indicate a success rate of implants of $96,9 \%$. Four implants were lost. $100 \%$ of patients were treated with overdentures, $60,9 \%$ with ball attachments and $39,1 \%$ with bar retained.

\footnotetext{
* Profesora Colaboradora Docente de Gerodontología. Facultad de Odontología. Universidad de Sevilla.

** Profesor Asociado de Odontología Integrada de Adultos. Facultad de Odontología. Profesor del Máster de Implantología Oral. Universidad de Sevilla.

*** Profesor Ssociado de Odontología Preventiva. Facultad de Odontología. Profesor del Máster de Implantología Oral. Universidad de Sevilla.

**** Profesor Titular de Odontología Integrada de Adultos. Facultad de Odontología. Director del Máster de Implantología Oral. Universidad de Sevilla.
} 
Conclusions. This study indicate that oral rehabilitation in the mandible of edentulous patients with overdentures supported by implants can achieve osseointegration and were loaded conventionally, early and immediately as a successful dental treatment.

KEY WORDS: Conventional loading, early loading, immediate loading, mandibular overdentures, osseointegration, implant dentistry.

Fecha de recepción: 25 de abril 2009.

Fecha de aceptación: 27 de abril 2009.

\section{INTRODUCCIÓN}

El tratamiento mediante una sobredentadura con implantes dentales ha revolucionado la terapéutica de los pacientes con edentulismo total que por problemas anatómicos, funcionales o económicos no pueden beneficiarse de una rehabilitación fija implantosoportada. La rehabilitación oral con una sobredentadura mediante la inserción de varios implantes en el paciente desdentado inferior ha representado en los últimos años una alternativa terapéutica odontológica muy favorable con un éxito elevado y una mejoría notable de la calidad de vida (1-9).

Clásicamente, se ha aceptado en el tratamiento con implantes dentales que se requiere un periodo de cicatrización libre de carga funcional (al menos 3 meses en la mandíbula y de 5 a 6 meses en el maxilar superior) para conseguir y mantener la oseointegración y evitar así la encapsulación fibrosa del implante (10). Este protocolo convencional de carga funcional, ha demostrado su eficacia clínica en sobredentaduras mandibulares (11-13). En este sentido, un estudio canadiense a largo plazo, durante un periodo medio de 15,5 años, valora 27 sobredentaduras con implantes Branemark. La supervivencia de los implantes fue del $93,1 \%$. Aunque fue necesario un mantenimiento adecuado de las sobredentaduras (ej. fracturas, rebases, etc), todos los pacientes revisados conservaron su prótesis durante el periodo de seguimiento clínico, ya que ninguna sobredentadura fue convertida en prótesis completa convencional ( $100 \%$ de éxito) (13).

Más recientemente, como consecuencia de un mejor conocimiento en los fenómenos biológicos de la oseointegración y de los resultados de la investigación experimental y clínica, diversos protocolos de carga precoz e inmediata se han ido incorporando al tratamiento con implantes demostrando un éxito elevado y mejorando las expectativas del paciente y del profe- sional al reducir el tiempo de duración del tratamiento (14-17). Esta carga precoz e inmediata de los implantes con sobredentaduras mandibulares ha sido posible, entre otros factores, por los avances en el diseño macroscópico de los implantes que han favorecido su inserción quirúrgica y estabilidad primaria, así como su conexión con los diversos aditamentos protésicos empleados en la realización de las sobredentaduras. Además el desarrollo de nuevas superficies rugosas (ej. chorreado de arena, grabado con ácido) que incrementan la unión al hueso del implante han mejorado los resultados de la carga precoz e inmediata (18-20).

Los protocolos de carga precoz e inmediata en la mandíbula de pacientes edéntulos han sido introducidos con un éxito similar a los estudios con carga convencional $(16,17)$. En este sentido, un estudio italiano compara dos grupos de 10 pacientes con sobredentaduras mandibulares sobre 4 implantes, con carga inmediata (2 días) y convencional (3-4 meses). A los 2 años, solamente un implante fracasó por periimplantitis en un paciente con carga convencional de su sobredentadura (21). Otro estudio neozelandés, compara dos grupos de 12 pacientes con sobredentaduras mandibulares sobre 2 implantes, con carga precoz (6 semanas) y convencional ( 3 meses). Después de un seguimiento de 2 años, no hubo ningún fracaso (22).

El objetivo del presente estudio era la realización de un análisis comparativo entre los diferentes tipos de carga funcional (inmediata, precoz y convencional) en sobredentaduras mandibulares.

\section{PACIENTESY MÉTODOS}

El presente estudio ha sido realizado en la Unidad Docente de Odontología Integrada de Adultos de la Facultad de Odontología y en el Máster de Implantología Oral de la Universidad de Sevilla. El estudio con- 
sistía en la rehabilitación con sobredentaduras con implantes orales en la mandíbula en pacientes edéntulos totales portadores de una prótesis completa.

Previamente a la realización del estudio, aquellos pacientes que padecían trastornos sistémicos graves que podían comprometer la oseointegración fueron excluidos del estudio (23). Todos los pacientes seleccionados eran adultos de ambos sexos.

Todos los pacientes seleccionados en el estudio fueron informados del protocolo del tratamiento con implantes, de los aspectos quirúrgicos y prostodóncicos, así como de la posible existencia de complicaciones y pérdida de implantes. Los pacientes autorizaron el tratamiento implantológico mediante un consentimiento informado. Antes del tratamiento, todos los pacientes fueron evaluados radiológicamente, con una ortopantomografía, y en los casos de reabsorción mandibular extrema con una tomografía computarizada.

El presente estudio no incluía la realización de técnicas quirúrgicas implantológicas complejas (ej. regeneración tisular, biomateriales, osteótomos, injertos óseos).

\section{Protocolos clínicos}

Se establecieron 3 grupos de pacientes con 3 protocolos implantológicos diferentes con respecto al tipo de implantes, cirugía y carga funcional (Tabla 1):

- Grupo I. Pacientes tratados con implantes roscados de superficie de titanio pulida IMTEC ${ }^{\circledR}$ (Imtec, Ardmore, EE.UU.), de forma no sumergida con colgajo e incisión supracrestal y carga funcional convencional a los 3 meses (12 semanas) de la inserción.

- Grupo II. Pacientes tratados con implantes roscados de superficie de titanio grabada con ácidos TSA Defcon $^{\circledR}$ (Impladent, Senmenat, España) de forma no sumergida con colgajo supracrestal y carga funcional precoz a las 6 semanas de la inserción de los implantes (Figs. 1-3).

- Grupo III. Pacientes tratados con implantes roscados de superficie de titanio grabada con arenado y grabado ácido SENDAX ${ }^{\circledR}$ (Imtec, Ardmore, EE.UU.) de forma no sumergida y sin colgajo y carga funcional inmediata en el mismo día de la inserción de los implantes.

Todos los pacientes fueron medicados, desde una hora antes de la cirugía, con un régimen antibiótico pre-

\begin{tabular}{|l|c|c|c|}
\hline \multicolumn{4}{|c|}{ TABLA 1. - DISTRIBUCIÓN DE LOS } \\
PACIENTES Y PROTOCOLOS CLÍNICOS \\
\hline & Grupo I & Grupo II & Grupo III \\
\hline$N^{\circ}$ pacientes & 8 & 5 & 20 \\
\hline$N^{\circ}$ implantes & 29 & 18 & 80 \\
\hline $\begin{array}{c}\text { Tipo de } \\
\text { implantes }\end{array}$ & IMTEC & Defcon & Sendax \\
\hline $\begin{array}{c}\text { Carga } \\
\text { funcional }\end{array}$ & Convencional & Precoz & Inmediata \\
\hline Retenedores & Barras & Barras & Bolas \\
\hline $\begin{array}{c}\text { Seguimiento } \\
\text { (meses) }\end{array}$ & 67,4 & 64,6 & 65,5 \\
\hline
\end{tabular}

ventivo (amoxicilina + clavulánico) durante una semana. Todos los pacientes recibieron anestesia local. Todos los pacientes realizaron el enjuague diario con clorhexidina durante los primeros 30 días.

El tiempo transcurrido de seguimiento clínico desde la carga funcional de todos los implantes fue al menos de 3 años (36 meses).

\section{Criterios de éxito}

Los criterios de éxito y supervivencia de los implantes fueron los recomendados por van Steenberghe et al (24). En este sentido, la supervivencia fue definida como la proporción de implantes permanentes en su localización original aunque no tengan valor clínico o cause efectos adversos.

\section{Análisis estadístico}

Se realizó una estadística descriptiva de los hallazgos clínicos del estudio, con referencia a las variables demográficas de los pacientes, las características de los implantes (supervivencia, el éxito, las complicaciones y pérdidas, tipo de diseño y superficie) así como de los diferentes tipos de carga funcional (convencional, precoz e inmediata) y de las restauraciones prostodóncicas (sobredentaduras). Todas las variables anteriores fueron analizadas estadísticamente. Las variables cualitativas fueron analizadas según el test de la chi-cuadrado, mientras que las variables 
cuantitativas fueron analizadas según el test de la varianza.

\section{RESULTADOS}

\section{Pacientes}

Treinta y tres pacientes edéntulos totales portadores de una prótesis completa (14 hombres y 19 mujeres) participaron en el estudio, con una edad media de 65, 1 años (rango: 42-83 años). No había diferencias significativas al relacionar el sexo y la edad de los pacientes (ANOVA, $p=0,1910)$. El 26,1\% $(n=12)$ de los pacientes tenían antecedentes periodontales, y el $17,4 \%$ eran fumadores $(n=8)$ (Tabla 1$)$.

\section{Implantes}

Se insertaron un total de 127 implantes (80 implantes Sendax, 29 implantes IMTEC y 18 implantes Defcon) en los correspondientes 33 pacientes, lo que representa una media de 3,8 implantes por paciente (Tabla 1).

Con respecto a la longitud: 4 implantes eran de $18 \mathrm{~mm}$; 7 , de $15 \mathrm{~mm}$; 59 , de $13 \mathrm{~mm}$; 10 , de $12 \mathrm{~mm} ; 40$, de $10 \mathrm{~mm}$, y 7 , de $8 \mathrm{~mm}$. Con respecto al diámetro: 80 implantes eran de $1,8 \mathrm{~mm} ; 24$, de $3,4 \mathrm{~mm}$, y 23 , de $3,75 \mathrm{~mm}$.

El 100\% de los implantes fueron insertados de forma diferida, tras un periodo mínimo de 6 meses después de la última extracción dental. Todos los implantes fueron insertados de forma no sumergida en una fase quirúrgica.

Entre las complicaciones hubo un caso de periimplantitis que no provocó la pérdida del implante (IMTEC), en un paciente con antecedentes periodontales y consumo de tabaco. Se perdieron un total de 4 implantes (2 Sendax y 2 Defcon) en 3 pacientes, lo que supone un índice de éxito del 96,9\%. No hubo diferencias significativas con respecto a los implantes perdidos en relación a los antecedentes periodontales, consumo de tabaco, número y tipos de implantes, tipo de carga funcional y sobredentadura (bolas o barra).

\section{Prostodoncia}

El $100 \%$ de los pacientes fueron rehabilitados con sobredentaduras mandibulares. El 60,9\% de los pacientes fueron rehabilitados con sobredentaduras median- te retención con bolas; mientras que el $39,1 \%$ con sobredentaduras con barras.

\section{Seguimiento clínico}

El seguimiento clínico medio de los pacientes estudiados ha sido de 67,5 meses (rango: 36-108 meses).

\section{DISCUSIÓN}

Con el advenimiento de la implantología oral, la posibilidad de realizar una terapéutica favorable del paciente desdentado inferior se ha convertido en una realidad odontológica. En este sentido, la experiencia clínica ha demostrado que la inserción de varios implantes pueden soportar una sobredentadura en la mandíbula edéntula con un éxito superior al $90 \%$. Además, las sobredentaduras con implantes oseointegrados pueden satisfacer al paciente edéntulo al proporcionar una estabilidad prostodóncica lo que mejora la función oral, desarrolla una estética aceptable, con un menor costo económico que la rehabilitación total fija implantosoportada $(4,6,7)$.

En este sentido, los resultados globales del presente estudio demuestran que mediante un adecuado protocolo diagnóstico, quirúrgico, prostodóncico y de mantenimiento, utilizando implantes con diversas superficies, insertados de forma no sumergida se puede conseguir una oseointegración adecuada y constituir una técnica predecible obteniéndose una supervivencia y éxito elevado de los implantes del $96,9 \%$, independientemente del tipo de carga funcional, como demuestra los estudios realizados en sobredentaduras mandibulares con implantes $(8,9,17)$.

La comparación de los resultados clínicos de los diferentes protocolos de carga funcional inmediata, precoz y convencional es importante porque su aplicación práctica puede reducir el periodo de tratamiento en beneficio de los pacientes. Sin embargo, frecuentemente, los resultados de los diferentes protocolos pueden no depender del tiempo de carga (diferida, pre$\mathrm{coz}$, inmediata) sino de otros factores como pueden ser la calidad del hueso, el consumo de tabaco, el bruxismo o de la combinación de todos estos factores (25, $26)$. En este estudio se ha intentado minimizar estos factores de riesgo ya que la inserción de los implantes se ha realizado en el sector anterior mandibular que ofrece una excelente calidad ósea y en pacientes con edentulismo total crónico donde las exodoncias corres- 
pondientes se realizaron más de 6 meses previo al tratamiento con implantes.

En el presente estudio se han realizado las sobredentaduras mandibulares sobre 2, 3 ó 4 implantes dependiendo, fundamentalmente, de las características orales del paciente, en relación a la cantidad de hueso disponible, ya que en el presente protocolo implantológico no se contemplaba la utilización de técnicas más complejas (ej. regeneración tisular, injertos óseos, factores de crecimiento) (Figs. 1-3).

Además de la estabilidad primaria de los implantes después de su inserción es importante conseguir un importante grado de oseointegración que posibilite el éxito a largo plazo del tratamiento con sobredentaduras mandibulares $(27,28)$. En este sentido, el diseño macro y microscópico de los implantes puede favorecer la unión hueso-implante. Aunque, los hallazgos clínicos con implantes con superficie pulida (ej. Branemark) han demostrado la eficacia en sobredentaduras mandibulares durante un largo periodo de tiempo $(1,2,13)$; sin embargo, las nuevas superficies rugosas por sustracción (ej. arenado y/o grabado ácido) han impulsado notablemente las expectativas de este tratamiento mediante la carga precoz e inmediata, ya que aceleran e intensifican la oseointegración $(14-16,18,19)$.

Después de la carga funcional de los implantes con sobredentaduras mandibulares, la pérdida de hueso marginal constituye un aspecto importante en la valoración del éxito a largo plazo. En este sentido, una revisión reciente demuestra que los implantes cargados de forma inmediata presentan una pérdida de hueso

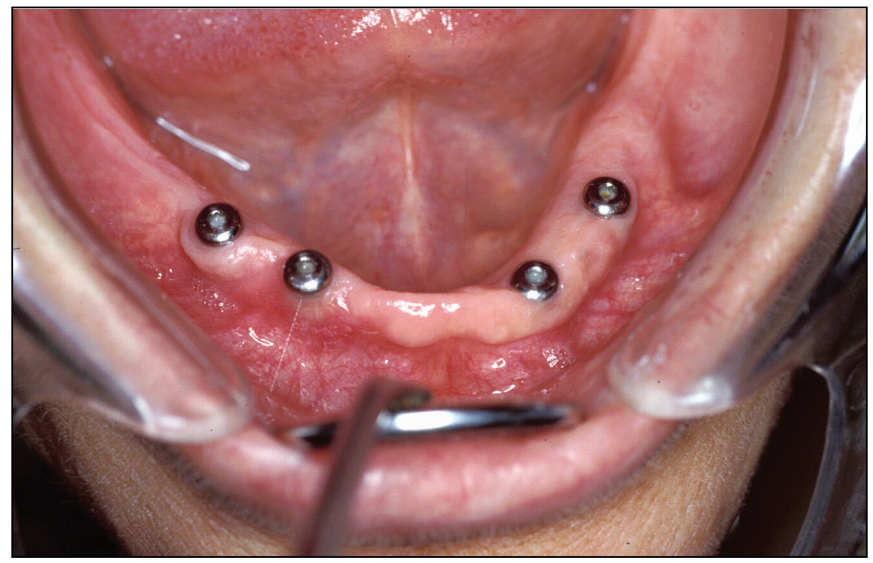

Fig 1: Aspecto clínico de los implantes insertados en la mandíbula.

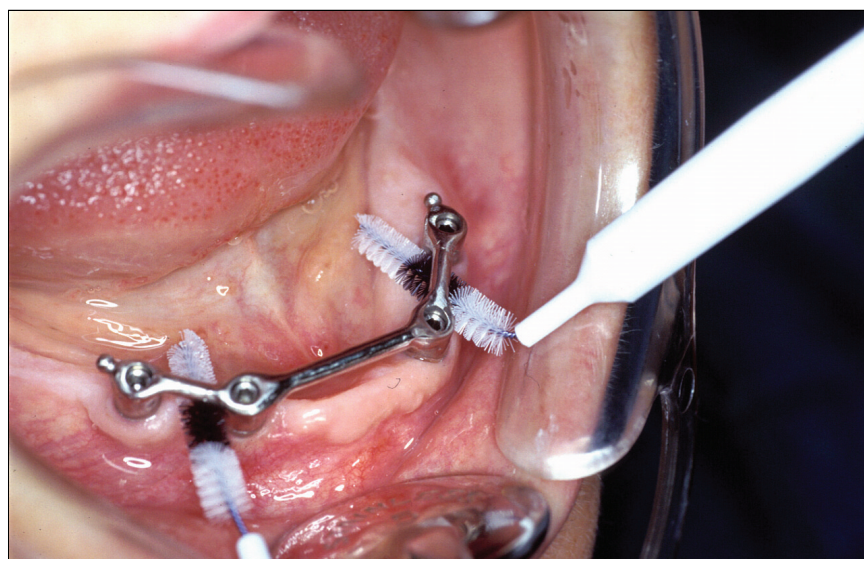

Fig. 2: Aspecto clínico de la barra de retención sobre los implantes. Importancia de la higiene oral.

marginal de 0,6 mm en los primeros 6 meses, y la misma proporción o más en el segundo año. Contrariamente, los implantes cargados de forma convencional presentan la misma respuesta ósea en el primer año, pero menor pérdida de hueso marginal en el segundo año (26). Los implantes cargados precozmente presentan menores pérdidas de hueso en ambos periodos de tiempo $(26,29)$.

Los parámetros periimplantarios suelen presentar una buena respuesta, independientemente del tipo de carga funcional (30). El índice gingival, el índice de sangrado periimplantario, el sondaje periimplantario y el índice de placa periimplantario han sido valorados sin diferencias significativas entre implantes cargados de forma convencional y precoz en sobredentaduras mandibulares durante un periodo de 2 años y puede estar

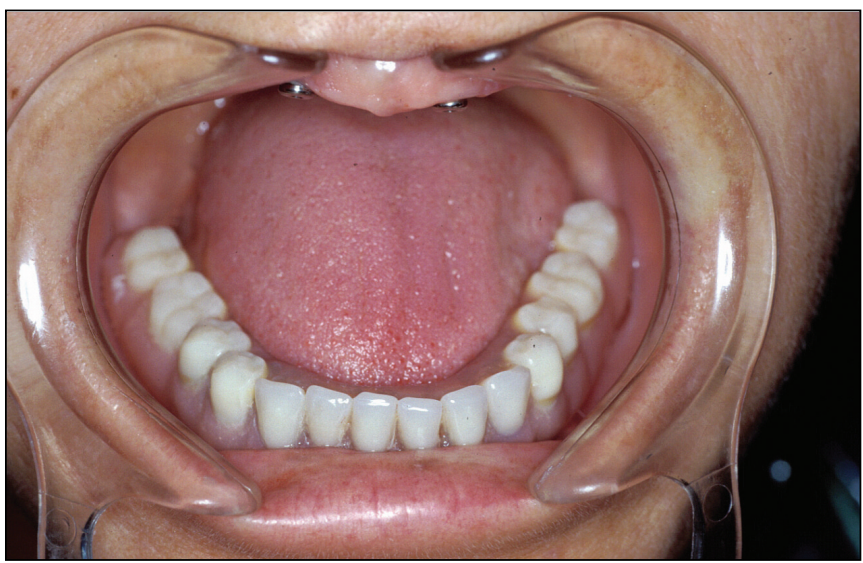

Fig. 3: Aspecto clínico de la sobredentadura mandibular 
más relacionados con los hábitos de higiene de los pacientes (Fig. 2) (30).

El presente estudio demuestra un éxito elevado del tratamiento con implantes $(96,9 \%)$ a los 5 años, ya que solamente se perdieron 4 implantes. Además, las complicaciones y fracasos fueron independientes del tipo de carga funcional y características de los implantes. La pérdida de los implantes fue resultado de complicaciones inmediatas que están relacionadas con el trauma quirúrgico de su inserción (31).

La oseointegración de los implantes permite su carga funcional y la correspondiente rehabilitación con sobredentaduras mandibulares. En este sentido, desde un punto de vista prostodóncico, los hallazgos clínicos de del presente estudio indican que en los diferentes protocolos de carga convencional, precoz e inmediata de los implantes orales, la rehabilitación posterior con sobredentaduras mandibulares en los pacientes edéntulos totales se ha conseguido un éxito del $100 \%$, ya que después de la fase quirúrgica ninguna sobredentadura ha tenido que ser sustituida o ha presentado complicaciones prostodóncicas importantes (Fig. 3).

Las sobredentaduras mandibulares implantosoportadas mejoran notablemente la calidad de vida de los pacientes edéntulos $(32,33)$. Desde un punto de vista funcional, el cambio en la capacidad masticatoria del paciente se incrementa, independiente de su carga funcional convencional o precoz sobre todo durante el primer año de tratamiento para disminuir y mantenerse posteriormente durante el segundo año, probablemente debido a una reabsorción ósea posterior. Este tipo de respuesta funcional es también observada en pacientes tratados con prótesis completa atornillada cargada de forma inmediata (25).

El mantenimiento a largo plazo de los pacientes con sobredentaduras mandibulares debe ser establecido, independientemente del tipo de carga funcional de los implantes. Los controles periódicos y los exámenes clínicos y radiológicos deben seguir un protocolo establecido $(5,13)$. Además, los diferentes tipos de anclajes o retenciones de las sobredentaduras mandibulares con implantes deben ser mantenidos para que su eficacia clínica sea permanente $(34,35)$. En el presente estudio, se han utilizado los 2 sistemas convencionales de anclajes en sobredentaduras, barra y bolas. Ambos sistemas son recomendables y han demostrado unos excelentes resultados a largo plazo (34). No obstante, una necesidad de mantenimiento frecuente es la activación o reubicación de los clips o ataches que retienen la sobredentadura en la supraestructura que es mayor conforme es menor el número de implantes relacionados. En este sentido, hasta el $70 \%$ de las sobredentaduras retenidas con 2 implantes pueden necesitar activación de los clips mientras que esta frecuencia desciende al $40 \%$ en las sobredentaduras con 3 ó 4 implantes (35).

\section{CONCLUSIONES}

El tratamiento con sobredentaduras mandibulares del paciente edéntulo con diferentes protocolos de carga funcional convencional, precoz e inmediata, ha demostrado su eficacia clínica a largo plazo como demuestra el presente estudio que según un estricto protocolo diagnóstico, quirúrgico, prostodóncico y de mantenimiento, pueden mantener la oseointegración y conseguir una rehabilitación funcional y estética del paciente con una elevada tasa de éxito.

\section{BIBLIOGRAFÍA}

1. Batenburg RHK, Meijer HJA, Raghoebar GM, Vissink A. Treatment concept for mandibular overdentures supported by endosseous implants: a literature review. Int J Oral Maxillofac Implants 1998;13:539-45.

2. Bergendal T, Engquist B. Implant-supported overdentures : a longitudinal prospective study. Int J Oral Maxillofac Implants 1998;13:253-62.

3. Mericske-Stern R. Treatment concepts of the edentulous jaw. En: Lang NP, Karring T, Lindhe J (eds.).Proceedings of the 3rd European Workshop on Periodontology. Implant Dentistry. Berlin: Quintessence. 1999. pag:376407.

4. Burns DR. Mandibular implant overdenture treatment: consensus and controversy. J Prosthod 2000;9:37-46.

5. Sadowsky SJ. Mandibular implant-retained overdentures: a literature review. J Prosthet Dent 2001;86: 468-73.

6. Feine JS, Carlsson GE. Implant overdentures. The standard of care for edentulous patients. Quintessence: Chicago. 2003.

7. Velasco E, Linares D, Velasco C, Monsalve L, Medel R. Las sobredentaduras con implantes oseointegrados en el paciente anciano. Av Perio Impl Oral 2003;15:25-33. 
8. Schwartz-Arad D, Kidron N, Dolev E. A long-term study of implants supporting overdentures as a model for implant succcess. J Periodontol 2005;76:1431-5.

9. Allen PF, Thomason JM, Jepson NJ, Nohl F, Smith DG, Ellis J. A randomised controlled trial of implant-retained mandibular overdentures. J Dent Res 2006;85:547-51.

10. Branemark PI, Zarb GA, Albrektsson T (eds.). Tissue integrated prostheses: osseointegration in clinical dentistry. Quintessence: Chicago. 1985.

11. Mericske-Stern R. Treatment outcomes with implantsupported overdentures: clinical considerations. J Prosthet Dent 1998;79:66-73.

12. Gotfredsen K, Holm B. Implant-supported mandibular overdenture retained with ball or bar attachments: a randomized prospective 5-year study. Int J Prosthodont 2000; 13:125-30.

13. Attard NJ, Zarb GA. Long-term treatment outcomes in edentulous patients with implant overdentures: the Toronto study. Int J Prosthodont 2004; 17:425-33.

14. Szmukler-Moncler S, Piatelli A, Favero GA, Dubruille JH. Considerations preliminary to the application of early and immediate loading protocols in dental implantology. Clin Oral Impl Res 2000;1 1:12-25.

15. Gapski R, Wang HL, Mascarenhas P, Lang NP. Critical review of immediate implant loading. Clin Oral Impl Res 2003; 14:515-27.

16. Chiapasco M. Early and immediate restorations and loading of implants in completely edentulous patients. Int J Oral Maxillofac Implants 2004; 19 (suppl):76-91.

17. Esposito M, Grusovin MG, Willings M, Coulthard P, Worthtington HV. The effectiveness of immediate, early and conventional loading of dental implants: a Cochrane systematic review of randomised controlled clinical trials. Int J Oral Maxillofac Implants 2007;22:893-904.

18. Velasco E, Pérez O, Medel R, Segura JJ, Torres R. Carga precoz de los implantes no sumergidos con superficie grabada con ácidos en sobredentaduras mandibulares. Arch Odontoestomatol 2003;19:308-16.

19. Velasco E, Segura JJ, Linares D, Medel R, Poyato M. La carga inmediata de los implantes transicionales en sobredentaduras mandibulares en adultos mayores. Av Perio Impl Oral 2004;16:107-13.
20. Meyer U, Wiesmann HP, Fillies T, Joos U. Early tissue reaction at the interface of immediately loaded dental implants. Int J Oral Maxillofac Implants 2003;18:48999.

21. Romeo E, Chiapasco M, Lazza A, Casentini P, Ghisolfi M, Iorio M, Vogel G. Implant-retained mandibular overdentures with ITI implants. A comparison of 2-year results between delayed and immediate loading. Clin Oral Impl Res 2002;13:495-501.

22. Payne AGT, Tawse-Smith A, Duncan WD, Kumara R. Conventional and early loading of unsplinted ITI implant supporting mandibular overdentures. Two year results of a prospective randomised clinical trial. Clin Oral Impl Res 2002;13:603-9.

23. Velasco E, Garcia A, Pérez O, Medel R, López J. La valoración médica del paciente implantológico oral. Factores de riesgo generales. Dentum 2006;6:13-8.

24. van Steenberghe D, Quirynen, Naert I. Survival and success rates with oral endosseous implants. En: Lang NP, Karring T, Lindhe J (eds.). Proceedings of the 3rd European Workshop on Periodontology. Implant Dentistry. Berlin: Quintessence. 1999. pag: 242-52.

25. De Smet E, Duyck J, Sloten JV, Jacobs R, Naert I. Timing of loading-immediate, early or delayed-in the outcome of implants in the edentulous mandible: prospective clinical trial. Int J Oral Maxillofac Implants 2007;22:58094.

26. KawaiY, Taylor JA. Effect of loading time on the success of complete mandibular titanium implant retained overdentures: a systematic review. Clin Oral Impl Res 2007;13:399-408.

27. Martinez H, Davarpanah M, Missika P, Celleti R, Lazzara R. Optimal implant stabilization in low density bone. Clin Oral Impl Res 2001;12:423-32.

28. Davies JE. Mechanisms of endosseous integration. Int J Prosthodont 1998;11:391-401.

29. Friberg B, Raghoebar GM, Grunet I, Hobkirk JA, Tepper G. A 5-year prospective multicenter study on 1-stage smooth-surface Branemark System implants with early loading in edentulous mandibles. Int J Oral Maxillofac Implants 2008;23:481-6.

30. Turkylmaz I, Tözüm TF, Tumer C, Ozbek EN. A 2-year clinical report of patients treated with two loading 
protocols for mandibular overdentures: early versus conventional loading. J Periodontol 2006;77:19982004.

31. Goodacre CJ, Kan KYK, Rungcharassaeng K. Clinical complications of osseointegrated implants. J Prosthet Dent 1999;81:537-52.

32. Bouma J, Boerrigter LM, van Oort RP, van Sonderen E, Boering G. Psychosocial effects of implant-retained overdentures. Int J Oral Maxillofac Implants 1997;12: 515-22.

33. Morais JA, Heydecke G, Pawliuk J, Lund JP, Feine JS. The effects of mandibular two-implant overdentures on nutrition in elderly edentulous individuals. J Dent Res 2003;82:53-8.
34. Mericske-Stern R, Taylor TD, Belser U. Management of the edentulous patient. Clin Oral Implant Res 2000; 11 (Suppl.): 108-25.

35. Payne AGT, Solomons YF. Mandibular implant-supported overdentures: a prospective evaluation of the burden of prosthodontic maintenance with 3 different attachment systems. Int J Prosthodont 2000;13:246-53.

\section{CORRESPONDENCIA}

Prof. Eugenio Velasco Ortega

Facultad de Odontología

C/ Avicena s/n

41003 Sevilla

e-mail: evelasco@us.es 